\title{
Factors Associated With Medication Errors Among Health Workers In University College Hospital, Nigeria.
}

\author{
Abosede Catherine Ojerinde RN, MSc (Nursing) ${ }^{1}$, Prisca Olabisi \\ Adejumo RN, $\mathrm{PhD}^{2}$ \\ ${ }^{I}$ Nurse Tutors Programme, University College Hospital, Ibadan, Nigeria. \\ ${ }^{2}$ Department of Nursing, University of Ibadan, Nigeria.
}

\begin{abstract}
A descriptive survey which purposefully selected respondents from the registered nurses and pharmacists working in the study center. A total number of 274 nurses and 59 pharmacists participated in the study ( $n=333$ ). Reliability coefficient was 0.6; Chi square and T-Test were applied to test hypotheses at $\alpha=0.05$ level of significance.

All the respondents ( $n=333$ ) indicated that exhaustion due to work pressure was the commonest cause of errors while 181 (66\%) of nurses and 9 (15.2\%) of pharmacists were previously involved in medication errors (MEs). Pharmacists ranked wrong labeling of patients' name (23.7\%) as the most common type of dispensing errors (Des) while overdose (30\%) was the commonest among nurses. There was no significant difference between the attitudes of nurses and pharmacists to MEs $(p=.044)$ but there was a significant difference between the rates of occurrence of MEs between nurses and pharmacists $(p=.000)$. Respondents further suggested double checking of prescription (32.2\%) and updating knowledge of pharmacology (16.9\%) as strategies for reducing MEs. 138 words
\end{abstract}

Key words: Dispensing error, administration error, medication, nurses, and pharmacists.

\section{Introduction}

Healthcare professionals, including nurses, strive in their day to day practice to effect optimal care for patients and clients and to ensure that they come to no harm. Medication error is defined as [1] any preventable event that may cause or lead to inappropriate medication use or patient harm while the medication is in the control of the health care professional, patient, or consumer. Such events may be related to professional practice, health care products, procedures, and systems, including prescribing order, communication, product labeling, packaging, and nomenclature, compounding, dispensing, distribution, administration, education, monitoring and use. The issue of proper medication use and safety is at the forefront of public health concerns globally and is likely to be larger from a global perspective. Errors can occur at any stage of the process; from medication selection and ordering, to order transcription, drug formulation, drug dispensing and administration. The role of the nurse in medication management has developed exponentially over time. However, the fundamentals of the nurse's role in medication management remain unchanged and nurses are expected to deliver and execute the highest standards of care and safety when it comes to medication management. Nurses represent the last safety check in the chain of events in medication management and therefore the final safeguard of patient wellbeing and potentially the difference between achieving the desired outcome and harming the patient. Dispensing is an integral part of the quality use of the medication and together with the patient counseling forms the core professional activities of a pharmacist. MEs that result in catastrophic events may result from combined efforts of "latent failures" in the system and "active failures" by individuals working in that system. Efforts are being made globally to establish systems capable of collecting accurate and relevant medication error data, which may provide valuable information needed to minimize MEs [2].

\subsection{Purpose of the study}

To determine the causes and factors contributing to medication administration/dispensing errors and the barriers to reporting errors among nurses and pharmacists in the University College Hospital Ibadan, Nigeria.

\subsection{Nature of the Problem}

The occurrence of MEs can compromise patient confidence in the health care system by exposing them to risk of adverse drug reactions, increase the costs of care, and even death [3]. The suffering is not only confined to patients, families, and health care agencies, even nurses, and pharmacists as well as other health care providers who are involved blame themselves for drug misadventures. They are worried and guilty, and the memory of the error stays with them for many years [4]. The Institute of Medicine report implicates [5] medication errors (MEs), at least in part as a direct cause between 44,000 and 98,000 patient deaths annually in 
the United States. The global burden of MEs results from all types of adverse events which includes prolong hospital stay, financial burden, disability, morbidity and mortality.

\subsubsection{Rationale for the study}

The results of this survey will provide information on factors influencing MEs in this environment and the development of standards to reduce the incidence of MEs. The knowledge also would assist with system redesign to reduce or eliminate barriers to reporting MEs ultimately ensuring highest quality patient care. Though the issue of MEs has attracted attention from several quarters of the world, there are some countries which do not have the essential ME monitoring systems and are oblivious to the problems created by them. However, efforts are now being taken in these countries, especially Nigeria and India through pharmacovigilance system, to gather information on adverse drug reactions. Stakeholders (health workers, patients, caregivers, hospital management) will be aware of the factors associated with MEs, why medication administration/ dispensing errors are not reported and how they can be reduced. Future researchers may also use this study as a reference and guide for future studies on medication administration (MAEs)/ dispensing errors (DEs) as there is paucity of local data in this area.

\subsubsection{Conceptual Framework (Fig 1)}

The concept of the theory of Human error [6] was utilized for this survey. There are three stages of cognitive processing for tasks namely, planning, storage, and execution, each cognitive stage has an associated form of error, execution stage is prone to slip error i.e incorrect execution of a planned action (e.g. misconception of command), storage stage is prone to lapses: incorrect omission of a stored, planned action (e.g. skipping a step on checklist) while planning stage is prone to mistakes i.e. plan not suitable for achieving the desired goal (e.g uncoordinated plan). Humans are inherently fallible and errors are inevitable therefore human psychology must not be ignored especially the skill-based, rule-based, and knowledge-based mistakes of human behavior at each level. Knowledge-based errors (e.g., inadequate knowledge of indications for medication use, allergic history, contraindication, etc. Rule-based errors i.e. rule violation (failure to follow accepted and wellestablished procedures). Skill-based or action-based in slips and memory lapses are unexplainable errors or errors due to forgetfulness.

The problem of human fallibility has two approaches, the person and the system. The person approach focuses on the errors of individuals i.e. forgetfulness, inattention, or moral weakness while the system approach concentrates on the conditions under which people work and tries to build defenses to avert errors or mitigate their effects. As a result, slips, lapses, and mistakes are all more common when situational factors divert attention. Situational factors include physiological factors like fatigue, exhaustion due to work stress, work overload, sleep deprivation, and psychological factors.

\section{Literature Review}

Medication management is a key component of nursing care provision and competence in this role by all health care workers especially nurses is crucial. There are numerous factors that exist in everyday nursing practice, all of which have the potential to contribute to MEs. In recent years, ME events received considerable attention because of its substantial mortality, morbidity, and additional health care costs. Minimal MEs can be achieved by identifying and adopting safety measures and where necessary facilitating a culture that will enhance patient safety, support nursing personnel and value the voices and concerns of those who speak out [7]. A dispensing error is a discrepancy between a prescription and the medicine that the pharmacy delivers to the patient or distributes to the ward on the basis of this prescription, including the dispensing of a medicine with inferior pharmaceutical or informational quality [8].

Reporting MEs could improve patient safety and provide valuable information for prevention of medication administration errors (MAEs) in the future. When hospitals identify ME trends and problem areas, they can prevent future errors and therefore reduce patient harm and injuries. At the moral level, hiding errors (especially those that are clinically significant) may also result in nurse patient fiduciary/trust relationship being undermined and ipsofacto the reputation of nursing profession as a whole [9].

\subsection{What are Medication errors?}

MEs are a challenge threatening health care system in every country, of the known ones, MAEs are the most common types [10]. They can be classified as a commission, which is giving the wrong medication, or an omission which usually means not administering or ordering a required medication. A potential error that could cause harm, but did not either by chance or by intervention, is classified as a near-miss event. All errors results in potentially negative outcomes for the client, including a near or actual death. The problems and sources of MEs are multidisciplinary and multifactorial. The action of one individual is rarely their solitary cause, rather a variety of contributing factors combine to cause incidents. A survey on registered nurses in medical-surgical 
units in a 700-bed community hospital in Florida [11]. Surveys were distributed to 92 full-time and part time Registered Nurses (RN) who administered medication at the hospital and 57 surveys were returned $(61.9 \%)$. Participants were asked to rank 10 perceived causes of MEs. The authors found that the top three perceived causes of MAEs were failure to compare the patient identification band with the administration record (35.7\%), nurse fatigue $(24.6 \%$,), and illegible handwriting by the prescriber $(12.3 \%)$.

\subsection{Factors associated with medication administration errors (MAEs)}

2.2.1Workload and staffing levels

These factors have been shown to affect the rate of MEs. [12]. An exploratory study [13] conducted to ascertain the opinions and recommendations of nurses in relation to factors in the work situation which contributed to MEs. A questionnaire containing open and closed ended questions was distributed to 175 nurses working in acute care settings. The findings were grouped into categories of contributory factors. 32 respondents cited contextual factors, including staffing problems, large patient workload and distractions whilst preparing medications and relief staff as being responsible for MEs.

\subsubsection{Single-nurse drug administration}

Several writers advocate that single-nurse drug administration does not increase the number of errors [14]. These authors describe a UK study of single-nurse drug administration over a 6-month period. A total of 18 nurses volunteered to take part in the scheme. These nurses underwent a series of lectures in pharmacology and a test of knowledge. Following this, seven nurses were deemed to have reached the required standard (test score above $90 \%$ ) and were invited to begin administering drugs alone. Over the 6-month period no errors were reported and staffs were of the opinion that single-nurse administration of drugs resulted in fewer errors.

\section{2.3.Distractions and interruptions}

Several authors have cited distractions as a factor in the occurrence of MEs [15]. Nurses administer medications in a sometimes chaotic environment, with many demands on their time. A fourteen observations studies [16] indicated that nurses cited interruptions as a significant contributor to MAEs. An interruption rate of 6.7 interruptions per hour to medication administration tasks was reported. Another similar study [17] reported the frequency of interruptions to 56 nurses' drug rounds in seven Italian surgical wards and revealed a rate of one interruption for every 3.2 drugs given, with the management of telephone calls reported to be a major source of interruptions. Similarly an observational [18] study among 30 nurses at Ohio State University Medical Center to determine relationships between work hours and MAEs and fatigue and MAEs. She found out that among all of the observed administrations, $16.6 \%(\mathrm{n}=91)$ of the medications given to patients had an interruption associated with them. Additionally, $1.1 \%$ of nurses ignored the interruption, $4.4 \%$ deferred the reason for the interruption until after she/he finished administering the medication(s) to the patients, and $94.5 \%$ of the nurses attended to the interruption when it occurred. Telephone calls were also reported to be a major source of interruptions. Other factors associated with MAEs identified in literature are mathematical skill of nurses, nurses' knowledge of medication/pharmacology, length of nursing shifts, length of nursing experience, nursing care and medication delivery level, policy and procedure.

\subsection{Factors associated with dispensing errors (DEs)}

\subsubsection{Drug labeling and packaging}

Confusion may occur with greater frequency during emergencies and urgencies. Five cases in which look-alike or sound-alike neonatal medication-dispensing errors occurred at the University of California San Diego Medical Center. A mix-up between neonatal and adult or pediatric products occurred in four of the five cases. Three of the five errors resulted in near misses with the potential to cause harm. The other two errors reached the patients but did not cause harm. The medication mix-ups involved adult and neonatal phytonadione injectable emulsion, sodium citrate injection and vancomycin-heparin combination injection, adult tetanusdiphtheria-acellular pertussis and infant diphtheria-tetanus-acellular pertussis (DTaP) vaccines, Haemophilus B and DTaP vaccines, and cisatracurium and vecuronium. Each error exposed weaknesses in the system of neonatal care [19].

\subsubsection{Working environment and conditions}

An unfavorable working environment tends to increase dispensing error rates. The designated area for drug dispensing should have adequate space and appropriate lighting, temperature, and humidity for comfortable work. Drugs should be stored in a way that facilitates the workflow, and furniture should be ergonomically distributed. The most significant cause of dispensing errors in community and institutional pharmacies is work overload.. Stress caused by imposing a maximum time limit for dispensing the prescription is also a significant factor. [20]. 


\subsubsection{Drug information}

Continuous technological development has led to frequent changes in information relating to drug use and safety. These changes have to be monitored by health professionals and applied in order for patients to benefit from them. Users of medical drugs may become allies in preventing MEs if they are well informed about the drugs being taken and can perceive errors not detected by health professionals. Patient counseling means additional safety against MEs. Studies have shown that $83 \%$ of errors were discovered during interviews with patients and corrected before they had left the pharmacy.[20] Other factors associated with DEs identified in literature include communication failures and drug information.

\subsection{Barriers to reporting Medication errors}

Voluntary medication error reporting systems rely on the ability and willingness of individual physicians, pharmacists, and nurses to detect and report errors as part of routine practice. Factors that reduce the potential for reporting MEs are management practices and professional cultures that punish an individual when errors are reported, even when the error is the result of poorly designed systems. Thus, there is significant underreporting of medication errors. The result is a significant decrease in the amount and quality of information that could be used to improve existing patient care systems and prevent future errors. [21]. A mail survey of 597 nurses conducted in one large medical center hospital in Taiwan to describe nurses' perceptions of barriers to reporting MAEs and to examine the relationship between the barriers to MAE reporting and cultural factors and nursing work environment. The survey revealed that the major perceived barrier was fear, Regression analysis showed that power hierarchy, face-saving concern, and work environment factors (e.g., quality management and peer relations) accounted for 54.6\% variance in the barriers. Age, educational background, working experience, experience of having made MAEs, and failure to report MAEs were not associated with the barriers. The association between the barriers and nursing work environment was negative and weak. [22].

\section{4 Managing Medication Errors}

Medication errors result from problematic processes, but their outcomes could range from minimal (or no) patient risk to life-threatening risk. The error classification should be based on the original order, standard medication dispensing and administration procedures, dosage forms available, acceptable deviation ranges, potential for adverse consequences and patient harm. Determination of the causes of MEs should be coupled with assessment process which should include programs to decrease the incidence of all MEs, and eliminating the causes of errors associated with greater levels of severity. There should be established mechanisms for tracking drugs or drug classes that are involved in errors. Quality improvement programs should provide guidance for patient support, staff counseling and education, and risk management process when a ME is detected. Risk management processes should include pharmacists, physicians, and nurses, in addition to risk management specialists, legal counsel, and others as appropriate. When appropriate, the supervisor and the staff members who were involved in the error should confer on how the error occurred and how its recurrence can be prevented.

Supervisors, department managers and appropriate committees should periodically review error reports, determine causes of errors and develop actions to prevent their recurrence (e.g., conduct organizational staff education, revise policies and procedures, or change facilities, equipment, or supplies). MEs should be reported to a national monitoring program so that the shared experiences of pharmacists, nurses, physicians and patients can contribute valuable educational services for the prevention of future errors.[23].

\section{Objectives}

To identify the frequency and types of MEs experienced by the respondents in the last 10 years.

To identify the causes of medication administration/ dispensing errors among respondents.

To identify the perceived barriers for non-reporting of MEs among respondents.

To determine the proportion of respondents who were previously involved in MEs.

To identify respondents' action to MEs after recognition.

To identify strategies to reduce MEs among nurses and pharmacists.

\subsection{Hypotheses}

There is no significant difference in the rate of occurrence of medication errors among nurses and pharmacists.

There is no significant difference between the attitudes of nurses and pharmacists to MEs.

4.1. Research Design

\section{Methods}

A descriptive study design was utilized for this study. 


\subsection{Study setting}

The study setting was the University College Hospital Ibadan (UCH). It is the first teaching hospital in Nigeria, established in 1951, a center of clinical excellence in Neuroscience, and currently the only hospital in West Africa with facility for Nuclear Medicine. The hospital is situated within the Ibadan North Local Government Area of Oyo State, Nigeria; it has 50 specialty and sub-specialty disciplines and runs 96 consultative clinics per week.. The bed space of the hospital is 850 with 1022 nurses while new capacities have been added in the private suite with 23 nurses and 36 beds.

\subsection{Study Population}

The study was intended to use 307 clinical nurses calculated through sample size determination formula with the attrition value of 28 (i.e. $10 \%$ of the sample size). 307 questionnaires were administered and 274 were suitable for analysis while 59 questionnaires were suitable for analysis out of 63 questionnaires administered to all the pharmacists working in the study center.

\subsection{Sampling Technique}

Incidental sampling was adopted for this study due to the fact that respondents run shift duties. Therefore this study utilized the following formula to calculate respondents from the sample frame of the Clinical Nursing Department, U C H (TABLE 1).

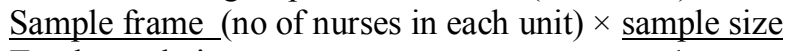

Total population

1

\subsection{Reliability}

The test-retest reliability showed a value of 0.570 (approximately 0.6 ) which indicated a moderate reliability.

\subsection{Validity}

The face and content validity of the instrument was ensured by comparing it with previous similar studies, also copies of the proposal was submitted to the supervisor for review, critique, and necessary amendment and correction was made.

\subsection{Ethical Considerations}

The study received approval from each participating departments and University's Institutional Review Board (IRB).i.e Ethical Review Committee of the University of Ibadan and University College Hospital Ibadan.

\subsection{Procedure for data collection}

Two research assistants were recruited for data collection and they were trained for two days. During the training, they were familiarized with the study settings, each question item, and the meaning of the desired information expected from each respondent. Data collection spanned a period of five weeks. The first set of questionnaire that addressed medication dispensing error was administered to all 63 pharmacists at the hospital and 60 were returned. Out of the 60 that responded one questionnaire was discarded because it was incomplete. The second questionnaire on medication administration was administered to 307 nurses in all the 20 units of the Clinical Nursing Department. The researcher cross-checked the returned questionnaires for completion and cleaning 274 were completed.

\subsection{Instrument}

Two sets of 58-item were used, the first set administered to the pharmacists addressed DEs while the second set administered to the nurses addressed MAEs. The respondents' attitudes and reasons for not reporting MEs were measured with a 5-point Likert's scale. The sections of the 58-item questionnaires were as follows: Section A: Socio-demographic data: sex, age, designation, work experience, and educational qualification -5 items.

Section B: Knowledge of pharmacology /dispensing processes-7 items

Section C: Reporting Medication errors-13 items

Section D: Causes of MAEs/DEs errors -15 items

Section E: Attitude/coping strategies to MEs - 15 items

Section F: Strategies to reduce MEs-3 items (one open-ended question)

\subsection{Data Analysis}

Completed data were analyzed using Statistical Package of Social Sciences (SPSS) version-17. The socio-demographic characteristics of respondents were assessed by running frequencies and percentages on the variables and results were presented in compressed table. In analyzing section F (open-ended questions) the 
researcher utilized a manually developed coding scheme for the emerged themes. The emerged strategies were manually grouped into categories.

\title{
V. Result
}

The socio-demographic characteristics of the participants showed that $44.1 \%$ of the pharmacists were within $21-30$ years while $55.9 \%$ of nurses were below 40 years, $57.7 \%$ of the pharmacists and $98.6 \%$ of the nurses were females respectively (table 2).

\section{Aim 1}

To identify the frequency and types of medication errors experienced by the respondents in the last 10 years

The finding showed that $52.5 \%$ of the pharmacists and $63.5 \%$ of the nurses make ME mistakes $1-2$ times in a month, while $5.1 \%$ and $2.9 \%$ make mistakes more than six times in a month of active practice respectively. The type of errors pharmacists had experienced in the last ten years was wrong labeling of patient name (23.7\%), followed by dosing errors (17\%) while 30\% of nurses had given drug overdose while $13.8 \%$ had administered under dose in the last ten years.

\section{$\operatorname{Aim} 2$}

To identify the causes of medication administration/ dispensing errors among respondents

Using a checklist of 14 specific causes of MEs (on a scale of minimum of 1 and maximum of 4 ) the result showed that the pharmacists ranked exhaustion due to work pressure $(\bar{x}=2.33 \pm 1.1)$ as the commonest cause of DEs followed by illegible doctor's prescription $(\bar{x}=2.25 \pm 1.1)$. The nurses also indicated that exhaustion due to work pressure $(\bar{x}=3.38 \pm 0.8)$ was the most common cause of MAEs followed by wrong dose calculation with $\bar{x}=3.29 \pm 0.8$. Other causes of MEs cited by respondents include poor labeling and packaging, illegible doctors' prescription, distraction, misinterpretation of prescription, confusion between two similar drug names and poor knowledge of drugs.(TABLE 3.1\& 3.2)

\section{$\operatorname{Aim} 3$}

To identify the perceived barriers for non-reporting of medication errors among respondents

The most common barrier to reporting MEs perceived by the pharmacists was fear of reprimand from those in authority (79.6\%) followed by fear of being recognized as incompetent $(69.5 \%)$. The commonest barrier perceived by the nurses was fear of being recognized as incompetent $(87.2 \%)$ followed by fear of reprimand from those in authority $(66.8 \%)$.

Aim 4 To determine the proportion of respondents who were previously involved in MEs; The result showed that $9(15.2 \%)$ of pharmacists and $181(66 \%)$ of nurses were previously involved in MEs.

\section{Aim 5}

To identify respondents' action to MEs after recognition

$20(33.9 \%)$ of the pharmacists will accept and apologize when medication error occur, $5(8.5 \%)$ will report it, while $1(1.7 \%)$ will cover it. Among the nurses $53(19.3 \%)$ will report it, $14(5.1 \%)$ will cover it up and give antidote, and $8(2.9 \%)$ will accept and apologize. $52.5 \%$ of pharmacists and $65.3 \%$ of nurses did not respond.

\begin{abstract}
Aim 6
To identify strategies to reduce MEs among nurses and pharmacists

The most frequent emerged strategy suggested by pharmacists was double checking prescription with reference book and a superior officer before dispensing (32.2\%), followed by adequate knowledge and drug update $(16.9 \%)$. While among the nurses continuous education on pharmacology $(29 \%)$ was the most frequent, followed by adhering to the 5-Rights of medication administration (17.5\%). Pharmacists' strategies (PS 1-13) and nurses' strategies (NS 1-13).(TABLE 4.1 and 4.2)
\end{abstract}

\section{Discussion}

The aim of the study was to determine the causes and factors contributing to MEs and why errors are not reported among pharmacists and nurses. According to Benner's Model of Skill Acquisition [24] majority of the nurses in this study were at expert level while majority of the pharmacist were either at the level of beginner, competence or proficiency (TABLE 2). An advanced beginner demonstrates acceptable performance, has gained prior experiences, and begins to formulate new ones to guide actions. Competent nurses are typically nurses 
with 2-3 years of experience on the job in the same area or in similar day-to-day situations. They are more aware of long-term goals, gains perspective from planning own actions based on conscious, abstract, and analytical thinking and help to achieve greater efficiency in the organization. A proficient nurse perceives and understands situations as a whole including its parts. They have more holistic understanding and improved decision-making. Furthermore proficient in experiences, what to expect in certain situations and how to modify plans when necessary. Nurse experts no longer rely on principles, rules, or guidelines to connect situations and determine actions. They have much more background of experience and have intuitive grasp of clinical situations. It may be assumed that the occurrence of medication errors should be lower among experts, but on the contrary a psychologist [6] argued that expertise alters one's level of cognitive regulation. He stated that with increasing expertise, the primary focus of control moves from knowledge-based towards skill-based levels. This suggests that the more skilled an individual is in carrying out a particular task, the more likely it is that his or her errors will take "strong-but-wrong" forms at the skill-based and rule-based levels of performance [6]. In view of these there is need for future studies to find out the relationship between skill acquisition and the occurrence of medication errors among health workers.

Result of respondents' frequencies of MEs in a month of active practice was lower compared to the finding of a six weeks prospective observational study [25] at the Aarhus University Hospital, Denmark. The report indicated dispensing errors at 22 out of $538(4 \%)$, and MAEs at 166 out of $412(41.2 \%)$ drugs administered. Instances cited by pharmacists in this study were dispensing wrong drug due to similar packaging (mixture of magnesium trisilicate for calmine lotion) and similar drug name/colour, giving an entirely different medicine (e.g. chlopropamode /metrpropamide, coarten / diclofenac, losartan / amilodipine). Instances cited by nurses were under dosage, (giving peadiatric dosage to adult), wrong drug, (gentamycin injection/digoxin, formalin/xylocaine), wrong calculation (incorrect dose of $\mathrm{Kcl}$ and $\mathrm{NaHCo3}$ ). Based on the above findings it is assumed that dosing errors cut across the respondents. Dosing error may be due to inadequate knowledge in pharmacology, poor mathematical skills non-availability of appropriate method of administering/dispensing paediatric dose, poor documentation, and technician's filling error. All the respondents in this study indicated that exhaustion due to work pressure was the main cause of MEs. This was supported by a survey [3] at the Obafemi Awolowo University, Ile-Ife. Nigeria, which indicated that all the pharmacists (100\%) who participated in their survey agreed that excess workload, was a major cause of DEs. In a related study [26] it was observed that the medication errors committed by nurses, physicians and pharmacists are $48.4 \%, 31.7 \%$ and $11.1 \%$ respectively. Results of this study assumed that the person mostly responsible for occurrence of medication errors is the nurse. Medicine is usually prescribed by the doctor and dispensed by the pharmacist, but responsibility for correct administration rests with the registered nurse. Nurses take responsibility for MA, as well as monitoring the prescribing practices of other professionals. They are the gate-keepers, maintaining active surveillance over the process on a continual basis [27]. With increase occurrence of MEs, nurses may felt vulnerable, and therefore, their medication administration practices may be motivated by factors such as fear and professional liability, instead of client safety. This could only be achieved if nurses actively drive change within both the clinical and research settings. It is imperative that clinically-based nurses contribute their expertise towards directing practice strategies, as well as driving research that examines the issue of MA

Using a checklist of 14 specific causes of MEs (on a scale of minimum of 1 and maximum of 4 ) .The finding of this study supported the affirmative statement (Reason, 2000) that humans are bound to commit errors. In the few root-cause analyses that have been performed, the most important causes of dispensing errors were related to organizational problems, such as shortages of staff and high workloads. This is consistent [28] with a finding which indicated that pharmacists rank work overload as the most significant factor contributing to errors. The responses of the nurses in this study agrees with the with the result of the survey at the Obafemi Awolowo University, Ile -Ife, Nigeria which showed that 785 (n-1000) nurses claimed that excess workload was a major cause of MAEs.[3] It is assumed that exhaustion due to workload is a major issue that needs to be explored among nurses and pharmacists working at the study center. The most common perceived barriers for non- reporting of MEs among the pharmacist and nurses were the fear of reprimand from those in authority. An interesting finding in this section of the study was the similar level of agreement with two of the reasons for not reporting MEs by the respondents. Reporting medication errors is fundamental to gathering information on such incidents, helps health professionals learn how to improve the medication-use process, and prevents or minimizes future incidents. For nurses, administering medication to patients is an important aspect of the daily routine which must be performed by a single nurse. Sometimes only the nurse knows about the medication error; hence, in those cases, voluntary reporting of medication errors to the department is the nurse's responsibility. When providers tell the truth, practitioners and patients share trust. This finding is also related to [29] a view which stated that respect for patient autonomy is paramount, as is the importance of veracity, fidelity, beneficence, and nonmaleficence which are all principles that orient reporting and disclosure policies.

Out of the proportion of nurses who participated in this stud $181(66 \%)$ had actually committed MAEs previously compared to $9(8.9 \%)$ pharmacist. Results of this study assumed that the person mostly responsible 
for occurrence of medication errors is the nurse. Therefore this study suggests that it is necessary to identify the contribution of medication errors from a nursing perspective. From the reactions of respondents at the recognition of error, it could be assumed that pharmacists' attitudes towards MEs were a little bit better than that of the nurses. Health care providers are typically embarrassed by their errors or omission and they may attempt to use defense mechanism by shifting the blame to someone or something else. In this study none of the pharmacists indicated they will deny error (s), quite a number of pharmacist indicated that they will accept and apologize, while very few of the nurses indicated this. It is a statement that acknowledges that an error has occurred and, as a consequence, expresses regret for having caused harm. An apology on the other hand may include a promise to refrain from engaging in similar conduct in the future. It may include an assurance that a full investigation will take place and the possibility for compensation for any harm that has been done. T-test analysis supported these findings that there was a significant difference between the attitudes of nurses and pharmacists in this study (TABLE 5).

Majority of the pharmacists suggested that there should be double-checking of prescription before dispensing (first pharmacists strategy i.e. PS1-). Quote: "Pharmacists should cross-check what they have dispensed before handing out medication to the patient. If it is possible the person who cross-check should be different from the person who dispense the drugs" (end of quote). Quote: "Bosses who are meant to supervise should not just sit down 'gisting' or doing other things not part of the scope of work". Majority of the nurses claimed that there was necessity for continuous education for nurses on pharmacology $79(29 \%)$ as the commonest theme, while 48 (17.5\%) agreed that nurses should follow strictly the 'Rights' of medication administration. Quote: 'MAEs can be reduced by having adequate knowledge of pharmacology and how to calculate medication dosage' (end of quote). Interruptions affect staff cognitively by interfering with working memory. The suggested interventions to reduce interruptions during medication related tasks which include the use of "protocol checklists", "interruption vests" and "No-Talk" signage, which have proved useful in reducing interruptions [30].

\section{Conclusion}

Each Registered Nurse is accountable for his or her practice. It is important to emphasize that nurses are pivotal in improving patient safety via error reporting. Patient safety will improve when systems effectively assure and improve safety, predicted on a culture in which the reporting of errors or near misses is considered valuable and positive actions lead to research and change for improvement, not blame. To avert underreporting of errors and to effectively learn from errors, administrators in healthcare institutions need to develop policies that support the routine reporting of errors, so that increased number of reports of actual errors and near misses are rewarded on an individual or unit basis. By easing the transition of an institution to a culture of safety. Medication errors should be a learning experience which could allow nurses to reflect on their own practice. The emphasis of errors should be shifted from the nurse to the situation in which it occurred as blaming the nurse was not considered to be very constructive. A comprehensive, collaborative, inter-professional solution will ultimately be required if the problem of medication errors is to be definitively addressed. Analysis of medication errors can lead to system improvement and reduced risk only if the errors are detected, reported, and used to design better patient care practices and systems.

\subsection{Limitations}

The study instrument relied on nurses' and pharmacists' perception, thus determination of the actual reasons errors occur or were not reported and is beyond the scope of this study.

It was conducted in a single University Teaching Hospital therefore results could be extrapolated and use as basis for further studies.

\subsection{Suggestions}

Health care professionals must provide adequate patient education about the appropriate use of their medications as part of any error prevention program. Proper education empowers the patient to participate in their health care and safeguard against errors. A multidisciplinary approach to solving the problem of medication errors is required which adopts an attitude of 'no blame' Internal quality control procedures for nurses and pharmacists. Regular update of healthcare professionals on pharmacology and drug update.

Observational study on factors associated with medication errors among nurses, doctors and pharmacists due to the fact that MEs are an important clinical problem that can occur at any stage of medication process. Direct observation of medication administration/dispensing errors to determine the effects of human factors on MEs. 


\section{Acknowledgement}

I am very much indebted to Dr. Bayo Adejumo (Dept of Psychology, University of Ibadan, Nigeria) and Dr Rose Ilesanmi for her guidance and contributions. I also appreciated Dr. Abimbola Oluwatosin and Dr. Modupe Oyetunde. (all from Dept of Nursing University of Ibadan, Nigeria). Finally I sincerely express my indebtedness to my children and my loving, faithful, enduring and understanding husband Eng. Kolawole Ojerinde.

Table 1: The Sample Frame and the Respondents

$\begin{array}{llll}\text { S/n } & \text { Unit } & \begin{array}{l}\text { Number } \\ \text { nurses }\end{array} & \begin{array}{l}\text { of } \\ \text { Respondents }\end{array} \\ \text { 1. } & \text { Accident and Emergency } & 55 & 55 / 1022 \times 307 / 1=16.5=17 \\ 2 . & \text { Main Theatre } & 73 & 73 / 1022 \times 307 / 1=21.9=22 \\ 3 . & \text { Accident \& Emergency (Theatre) } & 19 & 19 / 1022 \times 307 / 1=5.7=6 \\ 4 . & \text { Intensive Care Unit } & 48 & 48 / 1022 \times 307 / 1=14.4 \\ 5 . & \text { Labour Ward Complex } & 55 & 55 / 1022 \times 307 / 1=16.5 \\ 6 . & \text { Obstetrics \& Gynaecology } & 123 & 123 / 1022 \times 307 / 1=36.9=37 \\ 7 . & \text { Gynaecology Theatre } & 18 & 18 / 1022 \times 307 / 1=5.4 \\ 8 & \text { Obstetrics\&Gynaecology Theatre } & 03 & 03 / 1022 \times 307 / 1=0.9=1 \\ 9 . & \text { Central } & 06 & 06 / 1022 \times 307 / 1= \\ 10 & \text { Paediatrics } & 123 & 1.8=2 \\ 11 . & \text { Surgery } & 124 & 124 / 1022 \times 307 / 1=36.9=37 \\ 12 . & \text { Medicine } & 100 & 100 / 1022 \times 307 / 1=30 \\ 13 . & \text { Neuroscience } & 116 & 116 / 1022 \times 307 / 1=34.8=35 \\ 14 . & \text { Nephrology } & 65 & 65 / 1022 \times 307 / 1=19.5=20 \\ 15 . & \text { Out Patient Department } & 61 & 61 / 1022 \times 307 / 1=18.3 \\ 16 . & \text { Kola Daisi Foundation } & 11 & 11 / 1022 \times 307=3.3 \\ 17 . & \text { Hospice \& Palliative } & 06 & 06 / 1022 \times 307=1.8=2 \\ 18 . & \text { Nursing Audit } & 04 & 04 / 1022 \times 307=1.2 \\ 19 . & \text { Procedure Infection Control unit } & 06 & 06 / 1022 \times 307=1.8=2 \\ 20 . & \text { In-service Education \& Training unit } & 06 & 06 / 1022 \times 307=1.8=2 \\ \text { Total } & & \mathbf{1 , 0 2 2} & \mathbf{3 0 7}\end{array}$

Table 2: Percentage and frequency distribution of respondents' socio-demographic variables by age, sex, work experience and educational qualification.

\begin{tabular}{|c|c|c|c|c|c|}
\hline $\mathbf{S} / \mathbf{n}$ & Variables & Pharmacists & $N=59$ & Nurses & $\mathrm{N}=\mathbf{2 7 4}$ \\
\hline \multirow[t]{5}{*}{ A. } & Age & $\mathbf{N}$ & $\%$ & $\mathbf{N}$ & $\%$ \\
\hline & $21-30$ & 26 & 44.1 & 39 & 14.2 \\
\hline & $31-40$ & 23 & 38.9 & 153 & 55.9 \\
\hline & $41-50$ & 5 & 8.5 & 79 & 28.9 \\
\hline & $51-60$ & 5 & 8.5 & 3 & 1.0 \\
\hline \multirow[t]{3}{*}{ B. } & Sex & $\mathbf{N}$ & $\%$ & $\mathbf{N}$ & $\%$ \\
\hline & Male & 25 & 42.3 & 3 & 1.4 \\
\hline & Female & 34 & 57.7 & 270 & 98.6 \\
\hline \multirow[t]{8}{*}{$\mathrm{C}$} & $\begin{array}{l}\text { Years of work } \\
\text { experience }\end{array}$ & $\mathbf{N}$ & $\%$ & $\mathbf{N}$ & $\%$ \\
\hline & $1-5$ & 29 & 49.1 & 37 & 13.5 \\
\hline & $6-10$ & 13 & 22.0 & 92 & 33.6 \\
\hline & $11-15$ & 6 & 10.2 & 68 & 24.8 \\
\hline & $16-20$ & 3 & 5.1 & 45 & 16.4 \\
\hline & $21-25$ & 3 & 5.1 & 20 & 7.3 \\
\hline & $26-30$ & 3 & 5.1 & 6 & 2.2 \\
\hline & 31 above & 2 & 3.4 & 6 & 2.2 \\
\hline \multirow[t]{7}{*}{ D } & $\begin{array}{l}\text { Educational } \\
\text { qualification }\end{array}$ & $\mathbf{N}$ & $\%$ & $\mathrm{~N}$ & $\%$ \\
\hline & Diploma in nursing & - & - & 179 & 65.3 \\
\hline & $\begin{array}{l}\text { BSc (Phar) /BNSc } \\
\text { (Nursing) }\end{array}$ & 47 & 79.6 & 79 & 28.8 \\
\hline & $\mathrm{MSc}$ & 7 & 11.9 & 8 & 3 \\
\hline & PhD. & 1 & 1.7 & 5 & 1.8 \\
\hline & Post-doctoral & 2 & 3.4 & 2 & 0.7 \\
\hline & No response & 2 & 3.4 & 1 & 0.4 \\
\hline
\end{tabular}


Table 3.1 Causes of DEs among Pharmacists

Variables

Exhaustion due to work pressure

Illegible doctor's prescription

Humans are bound to commit error

Failure to check patient's name with prescription

Wrong prescription

Distraction

Wrong dose calculation

Absent-mindedness

Misinterpretation of prescription

Confusion between two similar terms

Poor labels/ packaging

Inadequate knowledge about the drug

Poor documentation

Wrong time of dispensing

\begin{tabular}{crr} 
N & Mean & \multicolumn{2}{c}{ 1. Deviation } \\
57 & 2.33 & 1.107 \\
56 & 2.25 & 1.083 \\
57 & 2.21 & 1.206 \\
56 & 2.18 & 1.114 \\
58 & 2.12 & 1.125 \\
57 & 2.11 & 1.080 \\
56 & 2.02 & 1.087 \\
58 & 2.02 & 1.051 \\
58 & 1.93 & 1.041 \\
57 & 1.91 & 1.057 \\
56 & 1.88 & 1.010 \\
57 & 1.75 & .912 \\
57 & 1.60 & 1.083 \\
58 & 1.55 & .940
\end{tabular}

Table 3.2 Causes of MAEs among Nurses

\section{Variables}

Exhaustion due to work pressure

Wrong dose calculation

Inadequate knowledge about the drug

Poor documentation

Wrong prescription

Poor labels/ packaging

Distraction

Failure to check patient's name with prescription

Misinterpretation of prescription

Confusion between two similar terms

Illegible prescription

Humans are bound to commit error

Absent-mindedness

Wrong time of administration
N

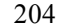

267

272

205

205

272

269

270

203

273

261

204

204

206
Mean

3.38

3.29

3.23

3.21

3.17

3.16

3.14

3.14

3.14

3.13

3.11

2.96

2.96

2.75
Std. Deviation

.876

.864

.914

.860

.934

.946

.940

.881

.900

.904

.972

.972

.948

Table 4.1. Qualitative analysis of Pharmacists response to strategies for reduction of Dispensing errors (DEs)

1. Double -checking prescription with reference book and superior officer

Category $\quad \mathbf{N} \quad \%$ Double -checking prescription with reference book and superior officer

PS-1 $19 \quad 32.2$

Adequate knowledge of pharmacology and drug update

Adequate staffing

Ensuring conducive environment

Legible prescription

Adequate client counseling

Adequate supervision by the immediate supervisor

Organizing interdepartmental seminars to create awareness of medication errors and fro prevention

Non-punitive measures for errors committed by pharmacists

Specialization in areas of medicine by pharmacists

Ward rounds for pharmacists

Counseling client on the importance of patience while waiting at the pharmacy

13. No response

Total

$\begin{array}{lll}\text { PS-2 } & 10 & 16.9\end{array}$

$\begin{array}{lll}\text { PS-3 } & 7 & 11.8\end{array}$

PS-4 $\quad 5 \quad 8.8$

$\begin{array}{lll}\text { PS-5 } & 4 & 7\end{array}$

PS-6 $\quad 3 \quad 5.0$

$\begin{array}{lll}\text { PS-7 } & 3 & 5.0\end{array}$

PS-8 $\quad 2 \quad 3.3$

$\begin{array}{lll}\text { PS-9 } & 2 & 3.3\end{array}$

PS-10 $\quad 1 \quad 1.6$

$\begin{array}{lll}\text { PS-11 } & 1 & 1.6\end{array}$

PS-12 1.6

PS-13 11.6

\section{'PS-Pharmacist Strategy}




\section{Table 4.2. Qualitative analysis of Nurses response to strategies for reduction of Medication} administration errors

\section{Nursing Strategy *NS}

\begin{tabular}{|c|c|c|c|c|}
\hline $\mathbf{S} / \mathbf{N}$ & Strategies & Category & $\mathbf{N}$ & $\%$ \\
\hline 1. & Continuous education on pharmacology for nurses & NS-1 & 79 & 29 \\
\hline 2. & Nurses should follow the 5-Rights of medication administration & NS-2 & 48 & 17.5 \\
\hline 3. & Avoid documentation of medication before administration & NS-3 & 26 & 9.5 \\
\hline 4. & Medication administration should be 2 -nurse procedure & NS-4 & 22 & 8.0 \\
\hline 5. & Avoid performing two procedures simultaneously & NS-11 & 15 & 5.4 \\
\hline 6. & Vigilance, patience, full concentration and avoid distraction & NS-5 & 14 & 5.1 \\
\hline 7. & Legible prescription & NS-7 & 12 & 4.3 \\
\hline 8. & Monitoring medication round by the nursing audit & NS-8 & 12 & 4.3 \\
\hline 9. & Maintain one nurse to $5 / 6$ patients & NS-9 & 11 & 4.0 \\
\hline 10. & Ensure adequate illumination on the ward & NS-10 & 9 & 3.2 \\
\hline 11. & Detected errors should be reported & NS-6 & 6 & 2.2 \\
\hline 12. & Correct labeling from pharmacy & NS-12 & 6 & 2.2 \\
\hline 13. & $\begin{array}{l}\text { Nurses should always clarify from colleague to differentiate trade and generic names of } \\
\text { drugs }\end{array}$ & NS-13 & 5 & 1.8 \\
\hline 14. & Through surveys & NS-14 & 1 & 0.4 \\
\hline 15. & Paying salary regularly & NS-15 & 1 & 0.4 \\
\hline \multirow[t]{2}{*}{16.} & No response & NS-16 & 7 & 7.4 \\
\hline & Total & 16 & 274 & 100 \\
\hline
\end{tabular}

Table 5. T-Test analysis of difference between the attitudes of respondents to MEs

\begin{tabular}{|l|l|l|l|l|l|l|l|}
\hline Variables & Mean & F & T & df & $\begin{array}{l}\text { mean } \\
\text { dif }\end{array}$ & $\begin{array}{l}\text { p value } \\
\text { sig }\end{array}$ & Decision \\
\hline $\begin{array}{l}\text { Nurses } \\
\text { Pharmacists }\end{array}$ & 32.65 & 16.125 & 0.6356 & 71.524 & 7.381 & .000 & $\begin{array}{l}\text { Significant } \\
\text { Null } \\
\text { hypothesis } \\
\text { rejected }\end{array}$ \\
\hline
\end{tabular}

\section{References}

[1]. National Coordinating Council for Medication Error Reporting and Prevention Retrieved on $3{ }^{\text {rd }}$ February, 2014 from http://www.nccmerp.org.

[2]. Balkrishnan, R, \&Furberg, C.D. Developing an optimal approach to global drug safety. journal of Internal Medicine, 250, 2009, 271-9. [PubMed]

[3]. Demehin, A.I., B \& Babalola, O.O., \& Erhun, W. O. Pharmacists and Nurses Perception of MedicationErrors in a Nigerian University Teaching Hospital. International Journal of health Research , 2008. Retrieved March 25, 2012 from http://.www.ijhr.org

[4]. Esi Owusu, A.R., \& While, A.. Medication errors: types, causes and impact on Nursing practice. British Journal of Nursing. 7(19), 2010, 380-5. Retrieved Nov.12, 2011 from http://www.ncbi.nlm.nih.gov/pumed .

[5]. Institute of Medicine. 2007 Crossing the Quality Chasm: A New Health System for the 21st Century. Washington DC, National Academy Press.

[6]. Reason, J. Human Error. Cambridge: Cambridge University Press 11, 2000 149-157. Retrieved Dec. 15, 2012 from http//ccform.com

[7]. Leufer, T \& Holdforth, J.C nurses key role represent the final safeguard against medication errors, World Irish Nursing Journal. 19(4), 2011, Retrieved $2^{\text {nd }}$ April, 2014 fromhttp://www.inmo.i.e/65.

[8]. Beso, A., Franklin, B. D. \& Berber, N. The frequency and potential causes of dispensing errors in a hospital pharmacy. Pharmacology World science 27, 2005 182-190.

[9]. Johnstone, M. J. \& Kanitsaki O. The ethics and practical importance of defining, distinguishing and disclosing nursing errors: a discussion paper. International Journal of Nursing Studie. (43), 2006, 367-376.

[10]. Sanghera, I.S., Franklin, B. D \& Dhillon, S. The attitudes and beliefs of healthcare professionals on the causes and reporting of medication errors in a UK Intensive care unit. Compilation of the Association of Anesthetists of Great Britain and Ireland, 62, 2007, 53-61.

[11]. Osborne J. Reducing medication errors: Strategies for nurses and healthcare organizations. 2005, Retrieved Nov 20, 2011 from http://www.vsigns.com

[12]. Roseman, C. \& Booker J.M. Workload and environmental factors in hospital medication errors. Journal of Nursing Research 44 (4), 1995 226-230.

[13]. Chenger P., Conklin D., Hirst S., Reimer H. \& Watson L. Nursing students in Alberta, their mathematical abilities. Advanced Journal of Nursing. (44), 1998, 17-22.

[14]. Jeanes, A. \& Taylor D. 1992. Stopping the drugs trolley. Nursing Times 88 (2), 1992 27-29.

[15]. Walters, J.A. Nurses' perceptions of reportable medication errors and factors that contribute to their occurrence. Applied Nursing Research. 5 (2), 1992, 86-88.

[16]. Biron, A., C. Loiselle, Work Interruptions and their contribution to medication administration Errors: An Evidence Review. Worldviews on Evidence-Based Nursing.Vol. 6 (2), 2009, 70-86. Retrieved Nov.10, 2011 from nursingcenter.com.

[17]. Palese, A., Sartor, A. Interruptions during nurses' drug rounds in surgical wards: observational study. Journal of Nursing Management. 17 (2), 2009, 185-192.

[18]. Bellebaum, K..L.The relationship between nurses' work hours, fatigue, and occurrence of medication administration errors in Ohio. PhD. Thesis. Pharmacy Dept. The Ohio State University, 2008.

[19]. Saubera, J.B, DeanL. M, Fiedelak, J \& Abraham, J.A. Origin of and solutions for neonatal medication dispensing errors. American Journal of Health System Pharmacy. 167(1), 2010, 49-57.

[20]. Cohen, H., Robinson, E. S., \& Mandrack, M. Getting to the root of medication errors. Nursing Times, (33), 2003 , 36-45. 
[21]. Wakefield B. J., Uden-Holman T, Wakefield D.,\& S Development and validation of the medication administration error reporting survey. Advances in patient safety from research to implementation, Programs, tools, and products. Surveys (4), 2005, Retrieved Dec. 12, 2011 from http://www.ahrq.gov/qual/advances/Vol4/Wakefield.

[22]. Chiang, H., \& Pepper, G.A. Barriers to nurses' reporting of medication administration errors in Taiwan. (39), 392-399, 2006 Retrieved Jan. 6, 2013, from orwww.ncbi.nlm.nih.gov .

[23]. Hughes, R.G.\& Blegen M.A.. Patient Safety and Quality: An Evidence-Based Handbook for Nurses. 2010.

[24]. Benner, P. Expertise in Nursing Practice. United States Springer Publishing Company 2001.

[25]. Lisby, M., Nielsen, L. \& Mainz, J. Errors in the medication process frequency, type, and potential, International Journal for Quality in Health Care, 17(1),2005, 15-22. Retrieved June, 2012 from http://intqhc.oxfordjournals.org/ 